\title{
Less invasive surgical treatment for aortic arch aneurysms in high-risk patients: A comparative study of hybrid thoracic endovascular aortic repair and conventional total arch replacement
}

\author{
Takashi Murashita, MD, Hitoshi Matsuda, MD, Keitaro Domae, MD, Yutaka Iba, MD, Hiroshi Tanaka, MD,
} Hiroaki Sasaki, MD, and Hitoshi Ogino, MD

\begin{abstract}
Objective: For aortic arch aneurysms, conventional total arch replacement has been the standard surgical option. In selected high-risk patients, we have attempted less invasive hybrid procedure involving supraaortic bypass and endovascular stent-graft placement. We review the early and midterm outcomes to clarify the impact of the hybrid procedure.
\end{abstract}

\begin{abstract}
Methods: Between October 2007 and December 2010, 27 patients were treated with the hybrid procedure. During the same period, 191 patients underwent elective conventional total arch replacement. On retrospective analysis, the hybrid procedure was feasible in 103 patients (hybrid feasible) and not feasible in 88 patients (hybrid impossible). Patients undergoing the hybrid procedure attained significantly higher additive (11.6 \pm 2.2 vs $9.5 \pm$ $2.4,10.3 \pm 2.8, P<.001, P=.044)$ and logistic $(31.1 \pm 14.1$ vs $18.8 \pm 12.6,23.7 \pm 16.0, P<.001, P=.047)$ European System for Cardiac Operative Risk Evaluation scores than hybrid-feasible and hybrid-impossible groups.
\end{abstract}

Results: Although the patients in the hybrid group had significantly higher risk, the early outcomes including mortality and morbidity were similar among the 3 groups, as were the 2-year survivals during the follow-up period: $85.9 \%$ for the hybrid group, $89.6 \%$ for the hybrid-feasible group, and $86.7 \%$ for the hybrid-impossible group $(P=.510, .850$, log-rank test). In the hybrid group, 2 patients required reintervention for type I endoleak.

Conclusions: The early and midterm outcomes of the hybrid procedure for aortic arch aneurysms were satisfactory. This procedure has the potential to be an alternative for conventional total arch replacement for high-risk patients. (J Thorac Cardiovasc Surg 2012;143:1007-13)

Conventional total arch replacement (TAR) using cerebral perfusion with hypothermia for brain protection has been the standard surgical option for aortic arch aneurysms, although it remains associated with some morbidity and mortality despite improved surgical technology. ${ }^{1}$ Cardiopulmonary bypass (CPB) is associated with a strong systemic inflammatory response and substantial myocardial injury, especially in high-risk subgroups. ${ }^{2}$ Moreover, deep hypothermic circulatory arrest can result in some permanent neurologic injury. ${ }^{3}$

Thoracic endovascular aortic repair (TEVAR) has recently been recognized as an alternative treatment for thoracic aortic aneurysms. This less invasive technique has also been used for aortic arch aneurysms using a supra-aortic bypass (hybrid arch TEVAR) with reportedly successful outcomes. ${ }^{4-9}$ Czerny and colleagues ${ }^{10}$ reported good midterm

\footnotetext{
From the Department of Cardiovascular Surgery, National Cerebral and Cardiovascular Center, Osaka, Japan.

Disclosures: Authors have nothing to disclose with regard to commercial support.

Received for publication March 23, 2011; revisions received May 16, 2011; accepted for publication June 27, 2011; available ahead of print July 25, 2011.

Address for reprints: Takashi Murashita, MD, 5-7-1 Fujishirodai, Suita, Osaka 5658565, Japan (E-mail: takashim@hsp.ncvc.go.jp).

$0022-5223 / \$ 36.00$

Copyright (c) 2012 by The American Association for Thoracic Surgery

doi:10.1016/j.jtcvs.2011.06.024
}

results with supra-aortic transpositions for extended endovascular repair of aortic arch aneurysms. Milewski and colleagues ${ }^{11}$ also reported the comparative study of elective open arch debranching with endovascular stent placement and conventional total and distal aortic arch reconstruction. They concluded that the hybrid procedure had a lower mortality in patients aged more than 75 years.

We used this technique for patients who had significantly high risk factors for conventional TAR. The aim of this study was to evaluate the early and midterm outcomes of hybrid arch TEVAR and to compare them with those of conventional TAR.

\section{MATERIAL AND METHODS \\ Patient Population}

In October 2007, we started performing hybrid arch TEVAR for aortic arch pathologies in selected high-risk patients. For these patients, conventional TAR was difficult because of some surgical risks, for example, advanced age and some significant comorbidities of cerebrovascular disease, left ventricular dysfunction, history of cardiac surgery, pulmonary dysfunction, chronic renal failure, severely compromised daily life, and short life expectancy due to malignancy. Between October 2007 and December 2010, we performed hybrid arch TEVAR in 27 patients (hybrid-repair) with high risks. All operations were performed electively. The early and midterm outcomes were reviewed.

During the same period, we electively performed conventional TAR in 191 patients. We retrospectively evaluated their aortic morphology and 


$\begin{array}{ll}\text { Abbreviations and Acronyms } \\ \text { BCA } & =\text { brachiocephalic artery } \\ \text { CPB } & =\text { cardiopulmonary bypass } \\ \text { CT } & =\text { computed tomography } \\ \text { ePTFE } & =\text { expanded polytetrafluoroethylene } \\ \text { euroSCORE } & =\text { European System for Cardiac } \\ & \text { Operative Risk Evaluation } \\ \text { LAxA } & =\text { left axillary artery } \\ \text { LCCA } & =\text { left common carotid artery } \\ \text { LSCA } & =\text { left subclavian artery } \\ \text { RAxA } & =\text { right axillary artery } \\ \text { RCCA } & =\text { right common carotid artery } \\ \text { TAR } & =\text { total arch replacement } \\ \text { TEVAR } & =\text { thoracic endovascular aortic repair }\end{array}$

background, such as risk factors and comorbidities. The hybrid arch TEVAR was not indicated in 88 patients primarily because of dilatation or dissection of proximal landing zone and sometimes younger age, infective aortic aneurysm, connective tissue disease, and need for concomitant cardiac procedures, such as valve replacement or coronary artery bypass grafting (hybrid-impossible group). For the remaining 103 patients, hybrid arch TEVAR or TAR was indicated in the assessment from the surgical aspect (hybrid-feasible group). We compared the outcomes of these 3 groups to justify this less-invasive procedure.

All patients underwent risk stratification according to European System for Cardiac Operative Risk Evaluation (euroSCORE) guidelines. ${ }^{12}$ Both additive and logistic values were collected because the logistic euroSCORE has been reported to be a better risk predictor for high-risk patients. ${ }^{13}$ Preoperative patient characteristics (Table 1) showed that patients in the hybrid-repair group were significantly older than patients in the hybrid-feasible group $(76.6 \pm 10.6$ years vs $71.3 \pm 8.0$ years, $P=.005)$. The significantly older age and higher rate of neurologic dysfunction ( $30 \%$ vs $2.9 \%, P<.001)$ resulted in a higher euroSCORE: The additive euroSCORE was $11.6 \pm 2.2$ vs $9.5 \pm 2.4, P<.001$, and the logistic euroSCORE was $31.1 \pm 14.1$ vs $18.8 \pm 12.6, P<.001$. Ischemic heart disease $(56 \%$ vs $18 \%, P<.001)$ was more prevalent in the hybrid arch TEVAR group. Patients undergoing hybrid arch TEVAR also had a higher euroSCORE than the hybrid-impossible group (additive euroSCORE, $10.3 \pm 2.8 ; P=.044$; logistic euroSCORE, $23.7 \pm 16.0 ; P=.047$ ).

The data analysis was approved by the institutional review board of the National Cerebral and Cardiovascular Center, and the board waived the need for patients' consent.

\section{Preoperative Evaluation}

Preoperative evaluation was made by multislice computed tomography (CT) scans to predict the required length of the proximal landing zone after the supra-aortic bypass because an adequate proximal landing zone is essential for successful stent-graft placement. Furthermore, these CT scans help to exclude major occlusive disease of the supra-aortic branches and the aorto-iliac axis for arterial access for stent-graft insertion.

We chose the fashion of supra-aortic bypass appropriately according to an adequate proximal landing zone and inflow artery. ${ }^{14}$ The details of the operative procedure are shown in Table 2 . The supra-aortic bypass was performed once the proximal landing zone had been established between zones 0 and 2 . To ensure an adequate proximal landing zone of more than $2 \mathrm{~cm}$ in length, we aggressively chose zone 1 instead of zone 2. In this setting, only extrathoracic supra-aortic bypasses were required and less invasiveness could be preserved. However, in 4 cases with a proximal landing length less than $2 \mathrm{~cm}$ for the zone 1 landing, we alternatively could not avoid choosing the zone 0 landing. For this, median sternotomy and side-clamp of the ascending aorta for the anastomosis of the inflow site were required. We indicate this procedure in patients with an intact ascending aorta who are expected to be healthy enough for the sternotomy but have risks for $\mathrm{CPB}$.

The distal landing site was between $\mathrm{T} 3$ and $\mathrm{T} 7$ in 21 patients and between $\mathrm{T} 8$ and $\mathrm{T} 11$ in 6 patients.

Subset analysis for each hybrid reconstruction is shown in Table 3. There was no significant difference in patients' characteristics among the 3 groups.

\section{Surgical Procedures of the Hybrid Arch Thoracic Endovascular Aortic Repair}

Zone 0. After a median sternotomy and systemic heparinization, the ascending aorta was partially clamped and a straight Dacron prosthesis (10-14 $\mathrm{mm}$ in diameter) was sewn in a side-to-end fashion. The brachiocephalic artery (BCA) and left common carotid artery (LCCA) were clamped and divided while the mean systemic blood pressure was increased to more than $80 \mathrm{~mm} \mathrm{Hg}$ and then reconstructed in an end-to-end fashion. The left subclavian artery (LSCA) was reconstructed in an end-to-end fashion near its origin or the left axillary artery (LAxA) was reconstructed in a side-to-end fashion at the axillar portion. The origin of the LSCA was closed by coil embolization after TEVAR. The clamp times for BCA and LCCA were 8.3 minutes (range, 7-10 minutes) and 7.5 minutes (range, 7-8 minutes), respectively.

Zone 1. The right common carotid artery (RCCA) or right axillary artery (RAxA) was chosen as the inflow artery. The RCCA and LCCA were exposed through the middle cervical incision, and the RAxA was exposed at the subclavicular incision. The LAxA was exposed at the supraclavicular incision when the RCCA was the inflow artery for the bypass and through the subclavicular incision when the RAxA was the inflow artery. After systemic heparinization, a branched-type 8-mm expanded polytetrafluoroethylene (ePTFE) graft was anastomosed. The clamp times for RCCA and LCCA were 12.1 minutes (range, 8-17 minutes) and 7.6 minutes (range, 5-10 minutes), respectively.

Zone 2. Both of the AxAs were exposed under the subclavicular incision. An ePTFE graft of $8 \mathrm{~mm}$ was sutured onto both AxAs.

The transfemoral approach was selected as the access route for stentgraft placement for 5 patients. The native external iliac artery was selected for the approach for 7 patients, and a conduit with an 8-mm or 10-mm Dacron graft placed at the origin of the external iliac artery for the access route was used for 15 patients.

The Gore TAG (WL Gore \& Associates, Inc, Flagstaff, Ariz) was used in 21 patients, the Talent (Medtronic Inc, Santa Rosa, Calif) was used in 3 patients, and a Matsui-Kitamura stent-graft (Kitamura Inc, Kanazawa, Japan) was used in 1 patient. Both the Gore TAG and the Talent devices were used in 2 patients. An average of 1.4 devices were used (range, 1-3). The completed status of the supra-aortic bypass and TEVAR placement is shown in Figures 1 to 3.

For prevention of supra-aortic graft occlusion, all patients were given low-dose aspirin. Patients who had severely diseased arteries or who had undergone reconstruction of the left vertebral artery were given Coumadin.

\section{Surgical Procedure of Conventional Total Arch Replacement}

All patients underwent a median sternotomy for the establishment of CPB by means of RAxA perfusion in addition to femoral or ascending aorta perfusion. ${ }^{15}$ Patients were cooled to a core temperature of $20^{\circ} \mathrm{C}$ to $28^{\circ} \mathrm{C}$. Antegrade selective cerebral perfusion was used for cerebral safety. In most cases, a distal aortic anastomosis was created with a stepwise technique, ${ }^{16}$ and for arch reconstruction, a 4-branched graft was attached to the stepwise graft. This was followed by antegrade aortic perfusion. After 
TABLE 1. Comparison of patients' characteristics

\begin{tabular}{|c|c|c|c|c|c|}
\hline & $\begin{array}{l}\text { Hybrid repair } \\
\quad(n=27)\end{array}$ & $\begin{array}{l}\text { Hybrid feasible } \\
\quad(n=103)\end{array}$ & $\begin{array}{l}\text { Hybrid impossible } \\
\qquad(\mathrm{n}=\mathbf{8 8})\end{array}$ & $\begin{array}{c}P \text { value } \\
\text { (repair vs feasible) }\end{array}$ & $\begin{array}{c}P \text { value } \\
\text { (repair vs impossible) }\end{array}$ \\
\hline Age $(y)$ & $76.6 \pm 10.6$ & $71.3 \pm 8.0$ & $73.3 \pm 8.9$ & .005 & .154 \\
\hline Male:female & $22: 5$ & $79: 24$ & $62: 26$ & .595 & .378 \\
\hline Additive euroSCORE & $11.6 \pm 2.2$ & $9.5 \pm 2.4$ & $10.3 \pm 2.8$ & $<.001$ & .044 \\
\hline Logistic euroSCORE & $31.1 \pm 14.1$ & $18.8 \pm 12.6$ & $23.7 \pm 16.0$ & $<.001$ & .047 \\
\hline Chronic pulmonary disease & $2(7.4 \%)$ & $15(15 \%)$ & $5(5.7 \%)$ & .509 & .895 \\
\hline Extracardiac arteriopathy & $13(48 \%)$ & $2(31 \%)$ & $8(9.1 \%)$ & .097 & $<.001$ \\
\hline Neurologic dysfunction & $8(30 \%)$ & $3(2.9 \%)$ & $5(5.7 \%)$ & $<.001$ & .002 \\
\hline History of cardiac surgery & $1(3.7 \%)$ & $9(8.7 \%)$ & $10(11 \%)$ & .640 & .418 \\
\hline Serum creatinine $>2.0 \mathrm{mg} / \mathrm{dL}$ & $4(15 \%)$ & $8(7.8 \%)$ & $7(8.0 \%)$ & .452 & .493 \\
\hline $\mathrm{LVEF}<50 \%$ & $4(15 \%)$ & $5(4.9 \%)$ & $14(16 \%)$ & .165 & .868 \\
\hline Ischemic heart disease & $15(56 \%)$ & $19(18 \%)$ & $37(42 \%)$ & $<.001$ & .311 \\
\hline
\end{tabular}

$L V E F$, Left ventricular ejection fraction.

the LSCA was reconstructed with a branch graft, rewarming was initiated. The proximal anastomosis was constructed above the sinotubular junction. Finally, the LCCA and BCA were reconstructed and each anastomosis was deaired before release of the distal clamp.

\section{Data Definitions}

This comprises a retrospective analysis of prospectively collected data. Patients' risk factors were defined according to the euroSCORE calculator, ${ }^{12}$ which defines chronic obstructive pulmonary disease as long-term use of bronchodilators, steroids for lung disease, or less than $70 \%$ of $1 \mathrm{sec}-$ ond forced expiratory volume rate. Extracardiac arteriopathy is defined as the presence of 1 or more claudications, carotid occlusions or greater than $50 \%$ stenosis, and previous or planned intervention on the abdominal, limb arteries, or carotids. Neurologic dysfunction is defined as a disease severely affecting ambulation or day-to-day functioning, left ventricle ejection fraction is calculated by using the findings of transthoracic echocardiography, and ischemic heart disease is defined as a history of coronary artery intervention, history of coronary artery bypass grafting, and more than $75 \%$ stenosis of coronary arteries.

For postoperative mortality and morbidity, stroke was defined as permanent neurologic deficit caused by brain ischemia, embolism, thrombosis, or hemorrhage, and transient neurologic deficit was defined as the presence of abnormalities such as delirium or convulsion from which the patients eventually recovered. Renal failure was defined as new need for hemodialysis. Graft infection was diagnosed by examining CT scans or purulence drained from the prosthesis.

\section{Statistical Analysis}

The patients were followed up at the outpatient clinic or with a telephone survey, and the follow-up was completed for all patients. The median follow-up duration was 7 months (range, 1-27 months). The continuous data in this study are expressed as mean \pm standard deviation and range. Survival and freedom from aorta-related events were calculated with the

TABLE 2. Details of supra-aortic bypass

\begin{tabular}{llr}
\hline & \multicolumn{1}{c}{ Supra-aortic bypass } & $\mathbf{n}$ \\
\hline Zone 0 & Asc - BCA - LCCA - LSCA (LAxA) & 3 \\
& Asc-RAxA - LCCA - LSCA (LAxA) & 1 \\
Zone 1 & RAxA - LCCA - LAxA & 11 \\
& RCCA - LCCA - LAxA & 7 \\
Zone 2 & LAxA - RCCA - RAxA & 1 \\
\hline
\end{tabular}

Asc, Ascending aorta.
Kaplan-Meier method. Statistical analysis was performed with StatView (SAS Institute, Inc, Cary, NC).

\section{RESULTS}

In the hybrid arch TEVAR group, there was 1 in-hospital death $(3.7 \%)$. Cerebellar infarction during the preceding RCCA-LCCA-LAxA bypass surgery developed in this patient, who had a shaggy aorta. In the staged TEVAR, multiple embolism of liver, spleen, spine, and legs also occurred. The patient died 5 months after the operation. In another patient, cerebral infarction that caused quadrantanopia was detected. Stroke was encountered in these 2 patients $(7.4 \%)$, and no transient neurologic deficit was observed in the hybrid arch TEVAR group.

No brain damage was caused by the short-time clamp of the BCA and common carotid artery. However, in 2 patients $(7.4 \%)$, including the patient described above, paraplegia occurred after TEVAR. Another patient had both distal arch and descending aortic aneurysms. Two stent grafts were placed to cover from zone 1 to T11. The motorevoked potentials weakened during the skin closure. Paraplegia was not relieved, although cerebrospinal fluid

TABLE 3. Subset analysis for each particular hybrid reconstruction

\begin{tabular}{lccc}
\hline & $\begin{array}{c}\text { Zone 0 } \\
(\mathbf{n = 4})\end{array}$ & $\begin{array}{c}\text { Zone 1 } \\
(\mathbf{n = 1 9 )}\end{array}$ & $\begin{array}{c}\text { Zone 2 } \\
(\mathbf{n = 4})\end{array}$ \\
\hline Age $(\mathrm{y})$ & $80.0 \pm 13.4$ & $75.2 \pm 10.7$ & $79.8 \pm 8.0$ \\
Male:female & $4: 0$ & $16: 3$ & $2: 2$ \\
Additive euroSCORE & $11.3 \pm 2.4$ & $11.4 \pm 2.1$ & $13.0 \pm 2.2$ \\
Logistic euroSCORE & $27.1 \pm 12.2$ & $30.4 \pm 13.7$ & $38.5 \pm 18.5$ \\
Chronic pulmonary disease & $0(0 \%)$ & $2(11 \%)$ & $0(0 \%)$ \\
Extracardiac arteriopathy & $2(50 \%)$ & $8(42 \%)$ & $3(75 \%)$ \\
Neurologic dysfunction & $1(25 \%)$ & $6(32 \%)$ & $1(25 \%)$ \\
History of cardiac surgery & $0(0 \%)$ & $1(5.3 \%)$ & $0(0 \%)$ \\
Serum creatinine $>2.0 \mathrm{mg} / \mathrm{dL}$ & $0(0 \%)$ & $3(16 \%)$ & $1(25 \%)$ \\
LVEF $<50 \%$ & $0(0 \%)$ & $3(16 \%)$ & $1(25 \%)$ \\
Ischemic heart disease & $3(75 \%)$ & $9(47 \%)$ & $3(75 \%)$ \\
\hline
\end{tabular}

$L V E F$, Left ventricular ejection fraction. 


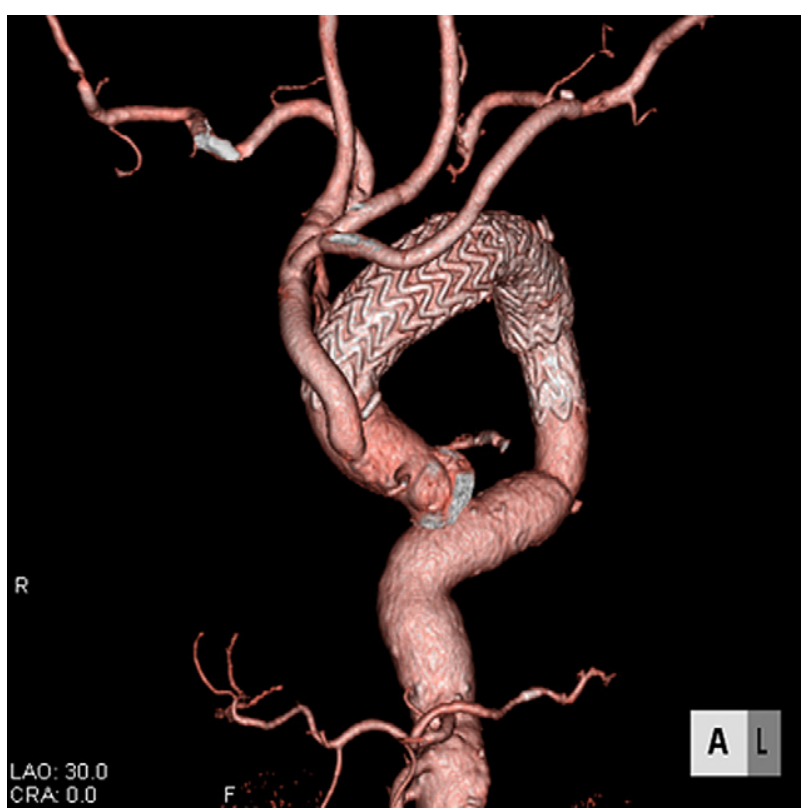

FIGURE 1. Postoperative 3-dimensional CT scan of hybrid arch TEVAR for zone 0 . Supra-aortic bypass was made from the ascending aorta to the BCA, LCCA, and LAxA.

drainage with steroid therapy was performed. In 3 patients $(11 \%)$, the injuries of the iliac arteries (the access route for TEVAR) were treated by graft replacement.

The median operation time including both bypass and TEVAR was 388 minutes (range, 212-540 minutes). No $\mathrm{CPB}$ was used. Eighteen of 27 patients $(67 \%)$ required transfusion.

The 2-year survival calculated by the Kaplan-Meier method was $85.9 \%$ in the hybrid arch TEVAR group. Two patients died of pneumonia 3 months and 4 months after TEVAR. In 2 patients, reinterventions for type I endoleak were necessary after TEVAR with additional supra-aortic bypasses and TEVAR. No occlusion of the supra-aortic bypass was detected during the following period. No proximal dissection of the aorta was detected after TEVAR.

An outcome comparison of the 3 groups is shown in Table 4 . The 30 -day mortality (none vs $1.9 \%$ vs $2.3 \%$, $P=.881, .959)$ and in-hospital mortality $(3.7 \%$ vs $7.8 \%$ vs $8.0 \%, P=.753, .744)$ were similar. The incidences of stroke did not differ, but stroke tended to be more severe after TAR and resulted in deterioration of patients' activity. The requirement of tracheostomy and postoperative renal insufficiency were similar. The length of stay in the intensive care unit was significantly shorter in the hybrid repair group $(1.6 \pm 1.3$ days vs $5.6 \pm 9.0$ days vs $7.0 \pm 9.8$ days, $P=.024, .006)$. The midterm survival after the discharge calculated by the Kaplan-Meier method is shown in Figure 4. There was no significant difference among the 3 groups $(P=.510, .850, \log$-rank test $)$.

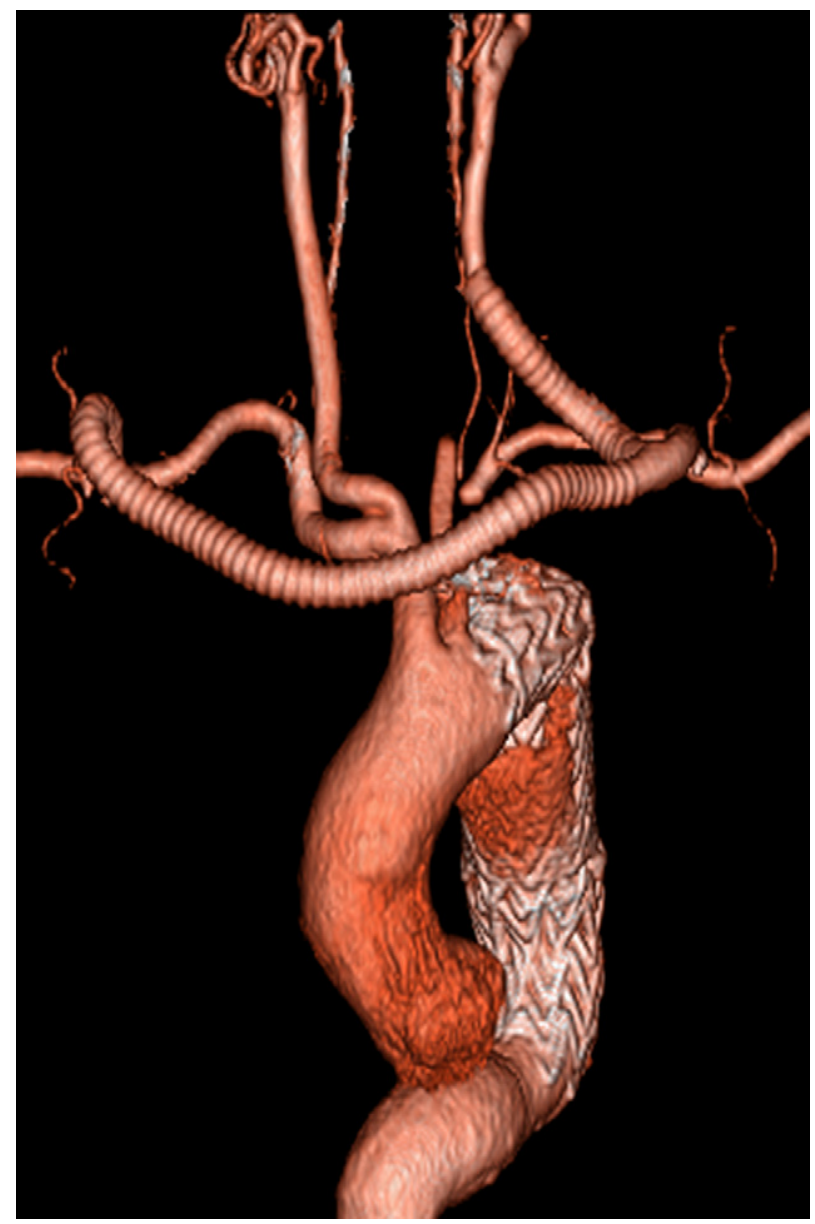

FIGURE 2. Postoperative 3-dimensional CT scan of hybrid arch TEVAR for zone 1. Supra-aortic bypass was made from the RAxA to the LCCA and LAxA.

\section{DISCUSSION}

The surgical outcome for aortic arch aneurysm has improved consistently. When balancing operative risk and anatomic suitability for less invasive endovascular treatment, TEVAR would be the most appropriate option for some patients who have complicated multiple medical comorbidities. ${ }^{17}$ Less invasive techniques for aortic arch repair with TEVAR are in demand because conventional TAR involves general anesthesia, median sternotomy, CPB, cerebral perfusion, and hypothermic circulatory arrest for systemic organ protection. Newer surgical alternatives that incorporate emerging endovascular techniques have the potential to transform the way we manage the aortic arch. In terms of TEVAR for arch aneurysms, there have been 2 approaches: the presented hybrid arch TEVAR and the TEVAR with a fenestrated or branched stent-graft. At the National Cerebral and Cardiovascular Center, a fenestrated or branched stent-graft cannot be used for TEVAR. The hybrid approach with the preceding supra-aortic bypass has been used as less invasive arch repair. 


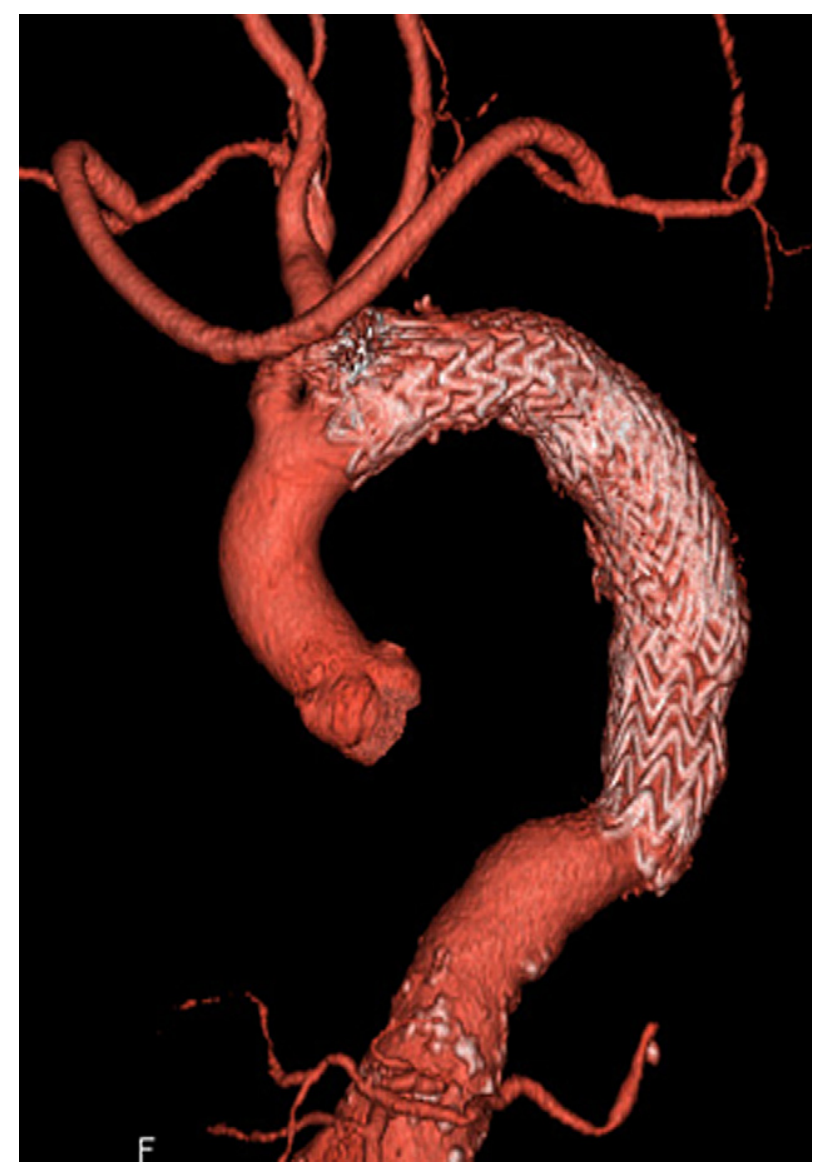

FIGURE 3. Postoperative 3-dimensional CT scan of hybrid arch TEVAR for zone 2. Supra-aortic bypass was made from the RAxA to the LAxA.

Similar studies have reported ${ }^{6-11}$ satisfying early and midterm outcomes of similar hybrid arch TEVAR using surgical debranching of the supra-aortic branches, followed by the endovascular repair. Our outcome was coincident to those of the other reports. However, we used this less invasive hybrid arch TEVAR for selected high-risk patients in situations in which conventional TAR had a satisfactory outcome with low mortality and cerebral morbidity. ${ }^{18}$ In the retrospective comparative study, the outcomes did not differ, although the hybrid arch TEVAR group had a significantly higher risk than the conventional TAR group. There have been few similar reports comparing the surgical outcome of the hybrid arch TEVAR and that of conventional TAR. Milewski and colleagues ${ }^{11}$ also reported the comparative study and concluded that the hybrid procedure may be a safe alternative to open repair; however, their treatment strategy was different from ours. They more aggressively created the proximal inflow anastomosis on the ascending aorta with the zone 0 approach in all cases via a median sternotomy with or without CPB. On the other hand, in the majority of our patients, the proximal landing zones were zone 1 or 2 where the patency of BCA was preserved and the extrathoracic supra-aortic bypass could be used. In 4 patients who required the proximal landing at the zone 0 , the inflow was made on the ascending aorta via a median sternotomy, because all of the patients have higher surgical risks. Weigang and colleagues ${ }^{19}$ also reported 26 cases of hybrid arch TEVAR. They performed an ascending aorta to bilateral carotid bypass using an inverse bifurcated prosthesis without $\mathrm{CPB}$ and deep hypothermic circulatory arrest. Four patients $(15 \%)$ died of perioperative cardiovascular adverse events. One of the main dangers is the construction of the end-to-side ascending aortic anastomosis. Szeto and colleagues $^{20}$ addressed the need for CPB before tangentially clamping the ascending aorta. We believe that if necessary, more aggressive zone 0 approaches might be justified when the ascending aorta is assumed to be safely side-clamped.

With regard to postoperative neurologic sequelae, less severe neurologic injury occurred in the hybrid arch TEVAR group than in the TAR group. Potential explanations for these findings are careful preoperative evaluation of the brain-supplying vessel, choice of the safe crossclamp and anastomosis site with CT scans and direct echocardiography, meticulous anastomosis, and short crossclamping

TABLE 4. Comparison of outcomes

\begin{tabular}{|c|c|c|c|c|c|}
\hline & $\begin{array}{l}\text { Hybrid repair } \\
(n=27)\end{array}$ & $\begin{array}{l}\text { Hybrid feasible } \\
\qquad(n=103)\end{array}$ & $\begin{array}{l}\text { Hybrid impossible } \\
\qquad(\mathrm{n}=\mathbf{8 8})\end{array}$ & $\begin{array}{c}P \text { value } \\
\text { (repair vs feasible) }\end{array}$ & $\begin{array}{c}P \text { value } \\
\text { (repair vs impossible) }\end{array}$ \\
\hline 30-d mortality & $0(0 \%)$ & $2(1.9 \%)$ & $2(2.3 \%)$ & .881 & .959 \\
\hline In-hospital mortality & $1(3.7 \%)$ & $8(7.8 \%)$ & $7(8.0 \%)$ & .753 & .744 \\
\hline Stroke & $2(7.4 \%)$ & $5(4.9 \%)$ & $4(4.5 \%)$ & .965 & .928 \\
\hline Transient neurologic deficit & $0(0 \%)$ & $8(7.8 \%)$ & $2(2.3 \%)$ & .296 & .959 \\
\hline Permanent paraplegia & $2(7.4 \%)$ & $0(0 \%)$ & $0(0 \%)$ & .057 & .083 \\
\hline Need for tracheostomy & $1(3.7 \%)$ & $5(4.9 \%)$ & $2(2.3 \%)$ & .794 & .778 \\
\hline Renal failure & $1(3.7 \%)$ & $1(1.0 \%)$ & $2(2.3 \%)$ & .881 & .778 \\
\hline Gastrointestinal bleeding & $1(3.7 \%)$ & $2(1.9 \%)$ & $1(1.1 \%)$ & .859 & .959 \\
\hline Reoperation for bleeding & $2(7.4 \%)$ & $3(2.9 \%)$ & $2(2.3 \%)$ & .604 & .501 \\
\hline Vascular injury & $3(11 \%)$ & $0(0 \%)$ & $0(0 \%)$ & .007 & .013 \\
\hline Graft infection & $0(0 \%)$ & $2(1.9 \%)$ & $2(2.3 \%)$ & .882 & .959 \\
\hline Intensive care unit stay (d) & $1.6 \pm 1.3$ & $5.6 \pm 9.0$ & $7.0 \pm 9.8$ & .024 & .006 \\
\hline
\end{tabular}




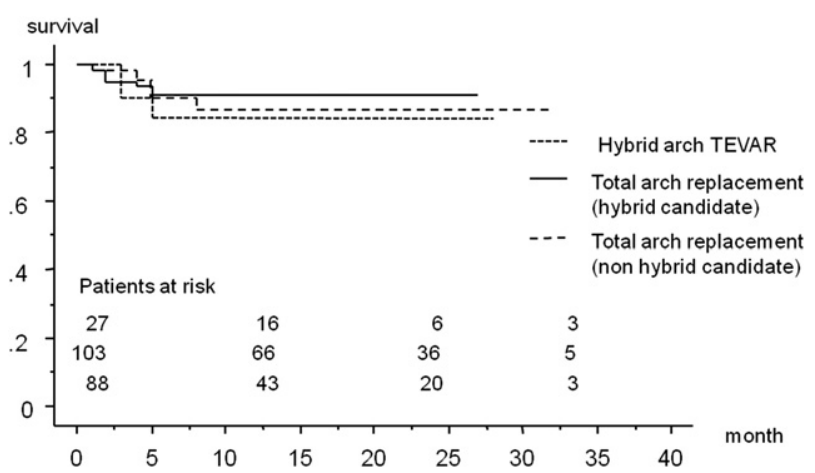

FIGURE 4. Kaplan-Meier survival curve. TEVAR, Thoracic endovascular aortic repair.

times of the brain-supplying vessels. Minor strokes, presumably due to embolism, were observed in only 2 patients. Without doubt, the risk of embolism is present in all these procedures, and careful manipulations and a crossclamp time short are mandatory.

Our data revealed that hybrid arch TEVAR is indicated for obviously "high-risk" patients. Whether the indication of this procedure will be extended to low- or mid-risk patients is the next issue to be investigated. There has been no report that shows long-term results of hybrid procedure. If the long-term durability of this procedure is established, the indication would be extended. Long-term durability can be greatly influenced by the presence of postoperative type I endoleak, required reintervention, and the patency of supraaortic bypass. In patients with type I endoleak, the following points are crucial in preventing it: (1) If the proximal landing at zone 2 is assumed to be difficult, adequate proximal landing zone should be obtained by making the landing at zone 1 and performing bypass surgery to the LCCA. (2) A stent-graft should be placed along the outer curvature of aortic arch to prevent the migration of the stent-graft. (3) When the BCA and LCCA have a common trunk (bovine aorta), a stent-graft should be placed covering approximately one third of the origin of common trunk after performing bypass surgery to the LCCA. Otherwise, new devices for the controlled deployment are assumed to have the possibility of reducing the defects of current commercially available devices, such as proximal neck wall apposition (the "bird's beak" effect) and aortic injury by the bare stent at the tip.

In the long-term, occlusion or thrombi associated with bypass grafting can lead to late neurologic complications. ${ }^{21}$ Byrne and colleagues ${ }^{22}$ analyzed 143 extra-anatomic procedures for carotid and subclavian reconstruction. Most bypass grafts were made of ePTFE and the 5-year patency rate was $92 \%$, indicating that artificial bypass grafts can result in excellent patency rates. We also used ePTFE grafts for most of our patients, and no graft occlusion was encountered during the follow-up period. However, we need further close observation of the patency of the supraaortic bypass in association with low-dose $(100 \mathrm{mg}$ ) aspirin administration.

There are some limitations to this study. First, the size of the cohort was small and the follow-up period was relatively short. Second, this was a single-institution, retrospective comparative study. There must be some potential bias in patient selection. Randomized controlled trials are thus desirable for accurate assessment of the best strategy for aortic arch pathologies.

\section{CONCLUSIONS}

The early and midterm outcomes of hybrid arch TEVAR for aortic arch aneurysm were satisfactory. Hybrid arch TEVAR has the potential to be a less invasive alternative for conventional TAR.

\section{References}

1. Kazui T, Washiyama N, Muhammad BA, Terada H, Yamashita K, Takinami M, et al. Total arch replacement using aortic arch branched grafts with the aid of antegrade selective cerebral perfusion. Ann Thorac Surg. 2000;70:3-8.

2. Czerny M, Baumer K, Kilo J, Lassnigg A, Hamwi A, Vukovich T, et al. Inflammatory response and myocardial injury following coronary artery bypass grafting with or without cardiopulmonary bypass. Eur J Cardiothorac Surg. 2000; 17:737-42.

3. Czerny M, Fleck T, Zimpfer D, Dworschak M, Hofmann W, Hutschala D, et al. Risk factors of mortality and permanent neurologic injury in patients undergoing ascending aortic and arch repair. J Thorac Cardiovasc Surg. 2003;26:5.

4. Criado FJ, Barnatan MF, Rizk Y, Clark NS, Wang C. Technical strategies to expand stent-graft applicability in the aortic arch and proximal descending thoracic aorta. J Endovasc Ther. 2002;9:1132-8.

5. Buth J, Penn O, Tielbeek A, Mersman M. Combined approach to stent-graft treatment of an aortic arch aneurysm. J Endovasc Surg. 1998;5:329-32.

6. Dambrin C, Marcheix B, Hollington L, Rousseau H. Surgical treatment of an aortic arch aneurysm without cardio-pulmonary bypass: endovascular stent-grafting after extra-anatomic bypass of supra-aortic vessels. Eur J Cardiothorac Surg. 2005;27:159-61.

7. Czerny M, Fleck T, Zimpfer D, Kilo J, Sandner D, Cejna M, et al. Combined repair of an aortic arch aneurysm by sequential transposition of the supraaortic branches and consecutive endovascular stent-graft placement. J Thorac Cardiovasc Surg. 2003;126:916-8.

8. Czerny M, Zimpfer D, Fleck T, Hofmann W, Schoder M, Cejna M, et al. Initial results after combined repair of aortic arch aneurysms by sequential transposition of the supra-aortic branches and consecutive endovascular stent-graft placement. Ann Thorac Surg. 2004;78:1256-60.

9. Gottardi R, Seitelberger R, Zimpfer D, Lammer J, Wolner E, Czerny M, et al. An alternative approach in treating an aortic arch aneurysm with an anatomic variant by supraaortic reconstruction and stent-graft placement. J Vasc Surg. 2005;42: 357-60.

10. Czerny M, Gottardi R, Zimpfer D, Schoder M, Grabenwoger M, Lammer J, et al. Mid-term results of supraaortic transpositions for extended endovascular repair of aortic arch pathologies. Eur J Cardiothorac Surg. 2007;31:623-7.

11. Milewski RK, Szeto WY, Pochettino A, Moser GW, Moeller P, Bavaria JE. Have hybrid procedures replaced open aortic arch reconstruction in high-risk patients? A comparative study of elective open arch debranching with endovascular stent graft placement and conventional elective open total and distal aortic arch reconstruction. J Thorac Cardiovasc Surg. 2010;140:590-7.

12. Nashef SAM, Roques F, Michel P, Gauducheau E, Lemeshow S, Salamon R, the Euro SCORE Study Group. European System for Cardiac Operative Risk Evaluation (EuroSCORE). Eur J Cardiothorac Surg. 1999;16:9-13.

13. Michel P, Roques F, Nashef SAM. Logistic or additive EuroSCORE for high-risk patients. Eur J Cardiothorac Surg. 2003;23:684-7.

14. Ishimaru S. Endografting of the aortic arch. J Endovasc Ther. 2004;11(Suppl 2): II62-71. 
15. Ogino H, Sasaki H, Minatoya K, Matsuda H, Tanaka H, Watanuki H, et al. Evolving arch surgery using integrated antegrade selective cerebral perfusion: impact of axillary artery perfusion. J Thorac Cardiovasc Surg. 2008;136: 641-8.

16. Ogino H, Ando M, Sasaki H, Minatoya K. Total arch replacement using a stepwise distal anastomosis for arch aneurysms with distal extension. Eur J Cardiothorac Surg. 2006;29:255-7.

17. Cronenwett JL. Endovascular aneurysm repair: important mid-term results. Lancet. 2005;365:2156-8

18. Sasaki H, Ogino H, Matsuda H, Minatoya K, Ando M, Kitamura S. Integrated total arch replacement using selective cerebral perfusion: a 6-year experience. Ann Thorac Surg. 2007;83:805-10.
19. Weigang E, Parker J, Czerny M, Peivandi AA, Dorweiler B, Beyersdorf F, et al Endovascular aortic arch repair after aortic arch de-branching. Ann Thorac Surg. 2009;87:603-7.

20. Szeto WY, Bavaria JE, Bowen FW, Woo EY, Fairman RM, Pochettino A. The hybrid total arch repair: brachiocephalic bypass and endovascular aortic arch stent graft placement. J Card Surg. 2007;22:97-102.

21. Weigang E, Luehr M, Harloff A, Euringer W, Etz CD, Szabó G, et al. Incidence of neurologic complications following overstenting of the left subclavian artery. Eur J Cardiothorac Surg. 2007;31:628-36.

22. Byrne J, Darling RC, Roddy SP, Mehta M, Paty PS, Kreienberg PB, et al. Long term outcome for extra-anatomic arch reconstruction. An analysis of 143 procedures. Eur J Vasc Surg. 2007;34:444-50. 